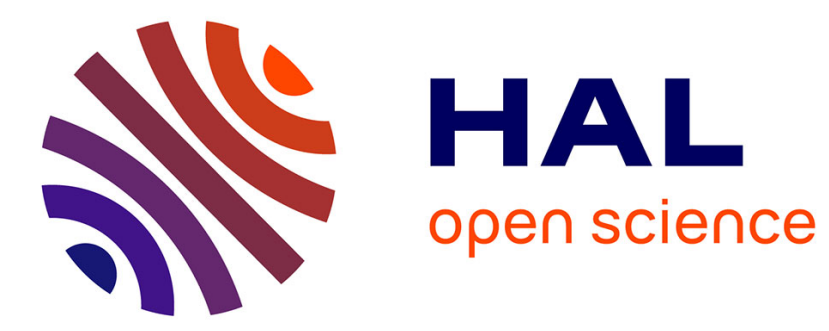

\title{
Endommagement des bétons à haute température
}

Ghassan Mounajed, Hocine Boussa, Abdellah Menou, Frédéric Grondin

\section{To cite this version:}

Ghassan Mounajed, Hocine Boussa, Abdellah Menou, Frédéric Grondin. Endommagement des bétons à haute température: Modélisation de la déformation thermique sous charge. Revue Européenne de Génie Civil, 2005, 9 (9-10), pp.1177-1190. 10.1080/17747120.2005.9692806 . hal-01007074

\section{HAL Id: hal-01007074 https://hal.science/hal-01007074}

Submitted on 21 Jan 2017

HAL is a multi-disciplinary open access archive for the deposit and dissemination of scientific research documents, whether they are published or not. The documents may come from teaching and research institutions in France or abroad, or from public or private research centers.
L'archive ouverte pluridisciplinaire HAL, est destinée au dépôt et à la diffusion de documents scientifiques de niveau recherche, publiés ou non, émanant des établissements d'enseignement et de recherche français ou étrangers, des laboratoires publics ou privés.

\section{(c)(1)}

Distributed under a Creative Commons Attribution| 4.0 International License 


\title{
Endommagement des bétons à haute température
}

\section{Modélisation de la déformation thermique sous charge}

\author{
Ghassan Mounajed* — Hocine Boussa* _ Abdellah Menou*** \\ Fréderic Grondin*****
}

* MOCAD - CSTB, Pôle Modélisation Calcul et Développement

Bât. 14, 84 avenue Jean Jaurès, F-77421 Marne-la-Vallée cedex 2

** LaSAGeC ISA BTP UPPA, 1 allée du parc Montaury, F-64600 Anglet

*** LM2S - CNRS UMR 7143

Université Pierre et Marie Curie

4, place Jussieu, F-75252 Paris cedex 5

RÉSUMÉ. Cet article entre dans le cadre de l'étude du comportement des ouvrages en béton exposés à l'incendie. Il a pour objectif de comprendre et de simuler les mécanismes d'endommagement et de déformation à haute température. Pour montrer l'effet structural des essais de type chauffage thermique sous charge, appelé "fluage thermique transitoire ", des simulations ont été menées avec le modèle Béton Numérique. Ces simulations montrent que la déformation appelée "fluage thermique transitoire ", constatée expérimentalement, est due essentiellement à l'endommagement thermique du béton lors du chauffage.

ABSTRACT. This paper deals with the study of concrete structures exposed to fire. The aim of this study is to investigate and to model the damage mechanisms of concrete after exposure to high temperature. Numerical simulations have been carried out in order to study the structural effect of confined concrete specimens when they are subjected to high temperature. Theses simulations show that the "transient thermal strain", noticed during experimental tests, is due to the thermal damage of concrete.

MOTS-CLÉS : endommagement thermique, modélisation multi-échelle, haute température, micro macro.

KEYWORDS: thermal damage, multi-scale model, high temperature, micro macro. 


\section{Introduction}

Les Bétons à Hautes Performances sont de plus en plus utilisés en France et dans les pays industrialisés. Une meilleure connaissance de ce matériau HP est acquise grâce aux différents travaux et projets de recherches menés depuis plusieurs années au niveau national et international. Ces travaux ont mis en évidence les caractéristiques intrinsèques et les performances très satisfaisantes de ce nouveau matériau. Malgré ses très bonnes performances à température ambiante, le BHP peut dans certains cas, présenter un comportement critique à haute température. L'analyse des effets des hautes températures sur sa microstructure est une étape nécessaire à la compréhension de son comportement thermomécanique.

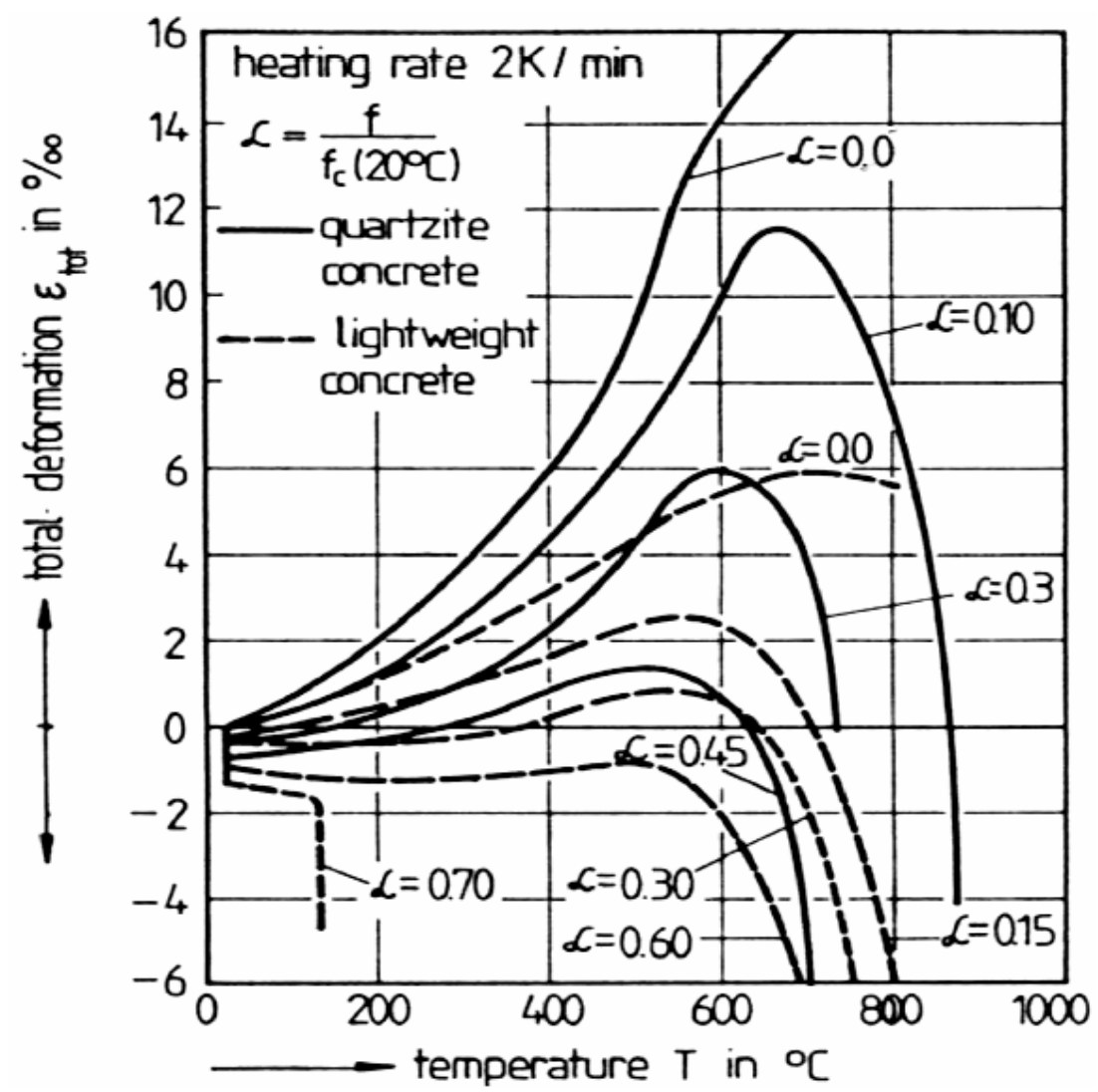

Figure 1. Evolution de la déformation thermique sous charge en fonction de la température pour différents niveaux de chargement et pour 2 types de béton (Schneider, 1988)

Différents travaux expérimentaux ont montré que la déformation thermique est fortement influencée par la présence simultanée d'un chargement mécanique pendant le chauffage. La figure 1 montre la déformation totale de spécimens en béton sollicités en compression à différents niveaux de chargement, allant de $0 \%$ à $60 \%$ de la résistance en compression, puis chauffés sous chargement constant (Schneider, 1988). Plusieurs auteurs expliquent l'allure de ces réponses par un phénomène appelé «fluage thermique transitoire » ou encore par «les déformations thermiques 
transitoires » qui caractérisent le comportement du béton lorsqu'il est simultanément soumis à un chargement mécanique et thermique (Khoury et al., 1985 ; Nechnech et al., 2002 ; Heinfling et al., 1997).

Pour étudier ce comportement, une nouvelle approche pour la modélisation de l'endommagement thermique est proposée dans cet article. Cette approche s'appuie sur le modèle "Béton Numérique» (Mounajed, 2002) et sur le modèle d'endommagement mécanique MODEV (Mounajed et al., 2003a ; Ung, 2003) du code aux éléments finis SYMPHONIE (Mounajed, 1991).

\section{Modélisation de l'endommagement du béton à haute température}

Le béton est un matériau multiphasique où chaque phase possède des caractéristiques mécaniques et physiques très différentes. Cette différence est encore plus importante sous chargement thermique car elle fait intervenir les coefficients de dilatation thermique de chaque phase dont la différence peut expliquer un endommagement prématuré non identifiable par une approche macroscopique homogène. En effet, l'expérience montre un écart important entre le coefficient de dilatation de la pâte de ciment et celui des granulats. Ce coefficient varie en fonction de la température, du type de ciment et du type de granulats (Diederichs et al., 1992).

L'endommagement thermique est décomposé en 2 parties représentant les principaux mécanismes qui conduisent à la dégradation du béton à haute température. Ces 2 parties seront décrites dans les sections 2.1 et 2.2 .

\subsection{Endommagement thermique d'origine mécanique dû aux déformations gênées}

Cet endommagement est lui-même la combinaison de deux endommagements.

Le premier est d'origine macromécanique dû aux déformations gênées à l'échelle macroscopique, comme celles dues au gradient de température ou au blocage des déplacements. Cet endommagement est modélisé d'une manière classique par le modèle MODEV qui sera présenté ultérieurement.

Le second, noté $d_{\text {micro, }}$ se manifeste à l'échelle mésoscopique, et est dû à la déformation micromécanique gênée, engendrée par la dilatation différentielle entre la pâte et les granulats. En effet, (Diederichs et al., 1992) ont constaté, en effectuant des mesures à haute température, un écart important entre le coefficient de dilatation de la pâte de ciment et celui des granulats. Cet endommagement a été étudié avec le modèle $\mathrm{BN}$, à l'échelle méso en prenant en compte pour les différentes phases (granulats, pâte de ciment) des caractéristiques mécaniques différentes (coefficient de dilatation thermique, modules, résistances...) et en supposant une adhérence 
parfaite entre la pâte et les granulats. Cette approche a confirmé que l'endommagement est lié à l'hétérogénéité du béton et qu'il augmente avec la température. La figure 2 montre les zones localement endommagées à $100{ }^{\circ} \mathrm{C}$. Il s'agit d'un échantillon en béton chauffé d'une manière homogène et en libre dilatation. L'interface pâte-granulats est la partie la plus touchée par l'élévation de température. L'évolution de l'endommagement est due à la dilatation différentielle pâte-granulats.

L'endommagement thermique d'origine mécanique (à l'échelle macro ou micro) est modélisé avec le modèle d'endommagement mécanique MODEV.

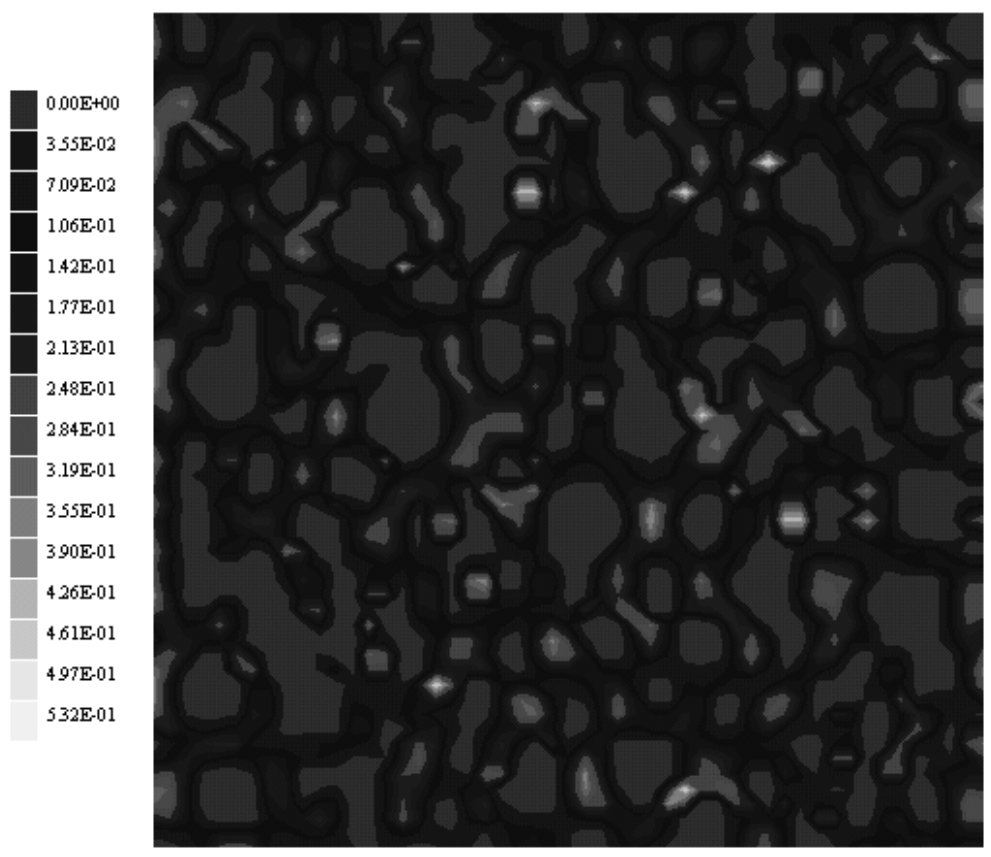

Figure 2. Zones localement endommagées (composante sphérique) à l'interface pâte granulats d'un échantillon en béton libre de se dilater et soumis à un champ thermique homogène $T=100{ }^{\circ} \mathrm{C}$

\subsection{Endommagement thermique d'origine physicochimique}

Cet endommagement est dû aux différentes transformations chimiques dans la pâte de ciment : déshydratation, perte de masse importante au-delà de $120^{\circ} \mathrm{C}$, et aux autres transformations chimiques.

Les travaux expérimentaux présentés dans (Menou, 2004) et (Mounajed et al., 2003b), montrent une baisse importante de la rigidité de la pâte de ciment en fonction de la température. Faute de résultats expérimentaux sur l'évolution du module des granulats à haute température, et sachant que les phénomènes physicochimiques interviennent surtout au-delà de $500{ }^{\circ} \mathrm{C}-700{ }^{\circ} \mathrm{C}$ selon le type de granulats, on postule que l'endommagement physicochimique noté $g$ a lieu essentiellement dans la pâte de ciment. Notons que le retrait de la pâte de ciment, le 
gonflement des phases cristallines, les variations dimensionnelles des granulats, dus aux transformations physicochimiques, sont pris en compte implicitement dans l'évolution des coefficients de dilatation thermique. Nous avons donc un couplage entre l'endommagement physicochimique et l'endommagement $d_{\text {micro. }}$.

L'évolution de l'endommagement physicochimique $g$ en fonction de la température a été identifiée à partir d'essais sur une pâte de ciment (Menou, 2004) en supposant celle-ci comme un matériau homogène à l'échelle de l'étude et en supposant la prépondérance des transformations physicochimiques dans la pâte de ciment.

\subsection{Présentation succincte du modèle d'endommagement mécanique MODEV}

Le modèle d'endommagement isotrope MODEV présente 2 modes d'endommagement: un endommagement par déformation déviatorique et un endommagement par extension sphérique.

Le modèle est basé sur la mécanique de l'endommagement et la thermodynamique des processus irréversibles. Il est formulé dans le cadre d'une transformation isotherme, un couplage endommagement-élasticité entre les deux modes de fissure a été considéré.

Un critère non symétrique en déformation a été utilisé. Par analogie avec la déformation équivalente au sens de Mazars (Mazars, 1984), deux nouvelles déformations équivalentes ont été introduites, traduisant respectivement le glissement local dans les microfissures et l'état d'extension hydrostatique. Elles sont respectivement calculées à partir des tenseurs de déformations déviatoriques et sphériques. On a ainsi deux valeurs d'endommagement, correspondant respectivement à chacun des deux mécanismes de dégradation. Chaque endommagement, ayant sa propre loi, évolue lorsque sa déformation équivalente atteint un certain seuil $K$.

$$
\begin{aligned}
& \boldsymbol{\varepsilon}=\boldsymbol{\varepsilon}^{d}+\boldsymbol{\varepsilon}^{s}=\boldsymbol{\varepsilon}^{d}+\varepsilon^{H} \mathbf{I} \\
& f\left(\boldsymbol{\varepsilon}^{s}, K_{s}\right)=\widetilde{\varepsilon}^{s}-K_{s}\left(d_{s}\right)=0 \\
& f\left(\boldsymbol{\varepsilon}^{d}, K_{d}\right)=\widetilde{\varepsilon}^{d}-K_{d}\left(d_{d}\right)=0
\end{aligned}
$$

$K_{s}(d)$ et $K_{d}(d)$ sont les fonctions seuil des endommagements sphérique et déviatorique. La déformation sphérique équivalente $\widetilde{\varepsilon}^{s}$ et la déformation déviatorique équivalente $\widetilde{\varepsilon}^{d}$ sont définies de la manière suivante : 


$$
\begin{aligned}
& \widetilde{\varepsilon}^{d}=\sqrt{\left(\varepsilon_{1}^{d}{ }_{1}\right)^{2}+\left(\varepsilon_{2}{ }_{2}\right)^{2}+\left(\varepsilon_{3}{ }_{3}\right)^{2}}+\alpha \varepsilon^{H} \\
& \widetilde{\varepsilon}^{S}=\varepsilon^{H}
\end{aligned}
$$

où $\varepsilon_{i}^{d}$ sont les déformations principales du tenseur de déformations déviatoriques, $\varepsilon^{H}$ est la déformation hydrostatique et $\alpha$ le coefficient de couplage sphériquedéviatorique, qui tient compte de l'influence de la consolidation du matériau par compression.

Le seuil initial d'endommagement est identifié par des essais élémentaires en traction uniaxiale et en cisaillement pur :

$$
\begin{aligned}
& \widetilde{\varepsilon}_{0}^{s}=K_{0 s}\left(d_{s}=0\right)=\frac{f_{t}}{3 \cdot E}(1-2 v) \\
& \widetilde{\varepsilon}_{0}^{d}=K_{0 d}\left(d_{d}=0\right)=\frac{\sqrt{2} f_{\text {cis }}}{2 G}=\frac{\sqrt{2} f_{t}}{E}(1+v)
\end{aligned}
$$

où $E, f_{t}, f_{c i s}, G, v$ : sont respectivement le module d'Young initial, la résistance en traction, la résistance au cisaillement, le module de cisaillement et le coefficient de Poisson. Le coefficient de Poisson est supposé constant.

Les équations donnant l'évolution des endommagements sphérique et déviatorique en fonction des déformations équivalentes respectives sont les suivantes :

$$
\begin{aligned}
& d_{s}=1-\frac{\widetilde{\varepsilon}_{0}^{s}}{\widetilde{\varepsilon}^{s}} \exp \left[-B_{t}\left(\widetilde{\varepsilon}^{s}-\widetilde{\varepsilon}_{0}^{s}\right)\right] \\
& d_{d}=1-\exp \left[-B_{c}\left(\widetilde{\varepsilon}^{d}-\widetilde{\varepsilon}_{0}^{d}\right)\right]
\end{aligned}
$$

où $B_{c}$ et $B_{t}$ sont des paramètres du modèle. La pente de la partie adoucissante $B_{t}$ de la loi de comportement en traction est liée à l'énergie de fissuration $G_{f}$ afin d'améliorer l'objectivité vis-à-vis du maillage (Hillerborg, 1976).

L'endommagement mécanique global $D$ est déduit par combinaison des endommagements correspondant à chaque mécanisme :

$$
1-D=\left(1-d_{S}\right)\left(1-d_{d}\right)
$$




\subsection{Modélisation avec le «Béton Numérique »}

Le modèle «Béton Numérique» $(\mathrm{BN})$ permet de prendre en compte l'hétérogénéité du béton. En effet, l'approche proposée permet de considérer le béton comme étant un matériau multiphasique avec $n$ phases de matériaux distribués spatialement d'une manière aléatoire. Ainsi, nous pouvons adopter les phases suivantes pour représenter un béton :

$1^{\text {re }}$ phase : squelette solide de la matrice ciment $M_{1}$;

$2^{\mathrm{e}}$ phase : une distribution aléatoire des pores avec la possibilité de décomposer cette phase en plusieurs sous-phases pour représenter des volumes et des natures différentes de pores $M_{2}, \ldots M_{x}$;

$3^{\mathrm{e}}$ phase : une distribution spatiale aléatoire des granulats avec la possibilité de décomposer cette phase en plusieurs sous-phases pour tenir compte des différentes tailles et différentes natures de granulats $M_{\chi}, M_{x+1}, \ldots$

Dans certains cas, et en particulier pour la modélisation des essais thermiques sous charge (Grondi et al., 2004), et pour des raisons pratiques, la phase des pores n'est pas modélisée explicitement. Nous avons considéré le béton comme un matériau à 2 phases homogènes : une phase pâte de ciment regroupant le squelette solide et les pores d'une part, et une phase granulats décomposée en $m$ sous-phases pour représenter les différents types de granulats d'autre part. La figure 3 présente la décomposition proposée.

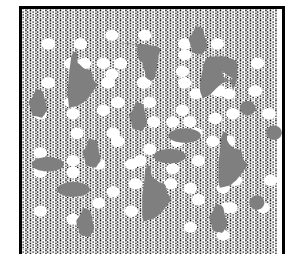

Béton

Numérique

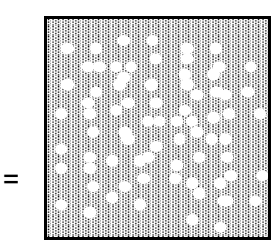

Pâte de ciment

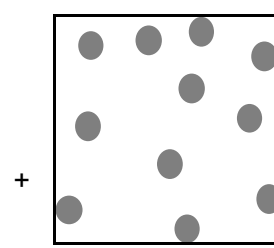

$1^{\text {re }}$ phase de + granulats

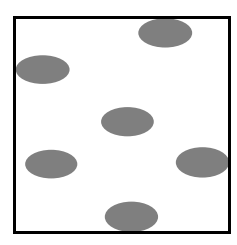

$2^{e}$ phase de granulats

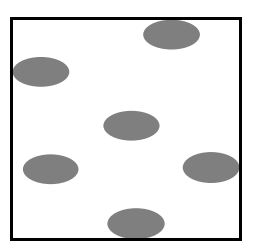

$N^{e}$ phase de granulats

Figure 3. Béton Numérique

Le modèle $\mathrm{BN}$ considère que chaque phase est caractérisée par un ensemble de paramètres mécaniques, thermiques et géométriques propres à la phase considérée. Ces paramètres sont regroupés dans le tableau 1.

La phase pâte de ciment et la phase granulats sont supposées avoir un comportement mécanique endommageable (MODEV). L'endommagement thermique d'origine physicochimique identifié expérimentalement est affecté à la phase pâte de ciment, sous forme d'une évolution du module en fonction de la température. Les effets liés à la taille du VER et à la répartition aléatoires des granulats ont été étudiés et présentés dans (Mounajed, 2002) et (Menou, 2004). 


\begin{tabular}{|l|}
\hline Le pourcentage du volume de la phase par rapport au volume total \\
\hline La taille unitaire du composant de la phase (diamètre du granulat) \\
\hline Modules de Young $E(\mathrm{MPa})$ et le coefficient du Poisson, $v$ \\
\hline Résistances à la traction de chaque phase $f_{t}$ \\
\hline Résistance à la compression \\
\hline Energie de fissuration $G_{f}$ \\
\hline Coefficient de dilatation thermique $\alpha_{\text {th }}$ \\
\hline Coefficient de couplage sphérique et déviatorique $\alpha_{\mathrm{s}}$ \\
\hline Ecrouissage de l'endommagement en compression $B_{c}$ \\
\hline Conductivité thermique \\
\hline Chaleur spécifique \\
\hline Masse volumique \\
\hline
\end{tabular}

Tableau 1. Caractéristiques mécaniques, thermiques et géométriques de la matrice cimentaire et des granulats introduites dans le Béton Numérique

\subsection{Etude expérimentale de l'endommagement thermique}

Un travail expérimental a été réalisé (Menou, 2004 ; Mounajed et al., 2003) avec un double objectif: identifier l'endommagement physicochimique nécessaire à la modélisation numérique et confronter le comportement expérimental des matériaux cimentaires à haute température aux simulations numériques. Cinq matériaux ont été testés : une pâte de ciment, un mortier à hautes performances, un béton ordinaire, un BHP à granulats calcaires et un BHP à granulats silico-calcaires.

Les matériaux ont été testés sur un bâti de flexion trois points selon les recommandations de la RILEM, de manière à obtenir des essais complètement stables. Pour chaque matériau, 3 éprouvettes $100 * 100 * 400 \mathrm{~mm}^{3}$ ont été testées en flexion 3 points jusqu'à la rupture. Les éprouvettes ont été testées à $20^{\circ} \mathrm{C}$ après avoir subi un cycle de chauffage-refroidissement avec une température de palier de $20,120,250$ ou $400{ }^{\circ} \mathrm{C}$. Une vitesse de $0,5^{\circ} \mathrm{C} / \mathrm{min}$ a été adoptée pour les phases de chauffage et de refroidissement. Cette vitesse a été optimisée par simulation (Menou, 2004) afin d'éviter l'endommagement mécanique par effet de gradient de température. De plus, la simulation de la répartition des températures pendant la phase de palier a montré qu'une durée de trois heures est nécessaire pour atteindre la quasi-uniformité des températures dans l'éprouvette, d'où le choix d'un palier de 3 heures.

Les résultats obtenus montrent une baisse très importante de la rigidité des matériaux après le cycle de chauffage-refroidissement. Les résultats montrent aussi une augmentation sensible de la ductilité et de l'énergie de fissuration $G_{f}$ à partir de $120^{\circ} \mathrm{C}$. 
L'étude expérimentale a aussi permis, en plus de l'obtention de l'évolution des principales caractéristiques thermomécaniques des matériaux cimentaires testés, d'alimenter le modèle d'endommagement thermique. A titre d'exemple, la figure 4 présente l'évolution de l'endommagement physicochimique $g$ dans la pâte de ciment en fonction de la température. L'endommagement mécanique global dans la pâte $D_{\text {pâte }}$ est déduit par combinaison des endommagements correspondant à chaque mécanisme :

$$
1-D_{\text {pâte }}=\left(1-d_{S, \text { pâte }}\right)\left(1-d_{d, p a ̂ t e}\right)\left(1-g_{\text {pâte }}\right)
$$

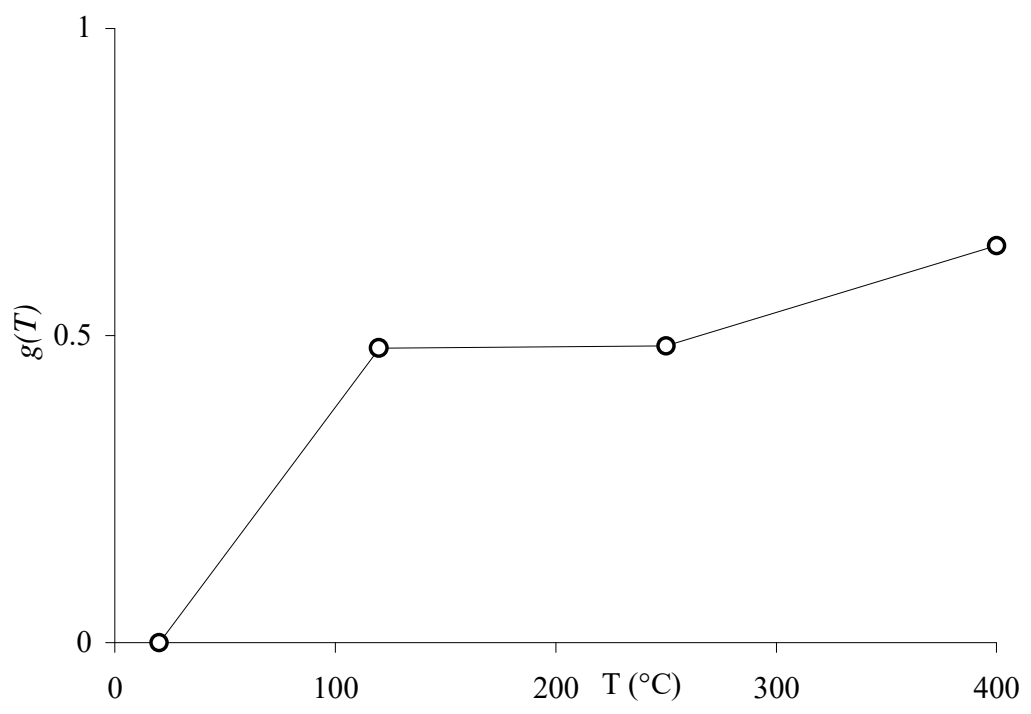

Figure 4. Identification expérimentale de l'endommagement thermique d'origine physicochimique de la pâte de ciment pour des températures allant de 20 à $400{ }^{\circ} \mathrm{C}$ (Menou, 2004)

\section{Modélisation de la déformation thermique sous charge de type FTT}

Les essais choisis pour la simulation sont issus des travaux expérimentaux réalisés par Holst (Holst, 1994). Il s'agit d'éprouvettes cylindriques de $132 \mathrm{~mm}$ de haut et de $68 \mathrm{~mm}$ de rayon, chargées et chauffées jusqu'à $800^{\circ} \mathrm{C}$ avec une vitesse de $4,98^{\circ} \mathrm{C} / \mathrm{min}$. Le chargement thermique est appliqué par l'intermédiaire d'une température imposée sur toutes les faces de l'éprouvette.

L'essai a été modélisé en mode axisymétrique en appliquant une charge qui représente l'équivalent de $0 \%, 20 \%, 40 \%$ et $60 \%$ de la résistance en compression de notre BHP (108 MPa). Cette charge est appliquée dès le début du chauffage et maintenue constante pendant toute la durée de l'essai. La figure 5 montre la géométrie, le maillage et les conditions aux limites adoptés pour le calcul.

La variable d'endommagement thermique d'origine physicochimique est prise en compte (Menou, 20004) ainsi que toutes les caractéristiques thermomécaniques du 
BHP trouvées dans (Holst, 1994). Pour utiliser le modèle Béton Numérique, il est également nécessaire d'avoir les caractéristiques mécaniques de chacune des phases (matrice cimentaire et granulats). Faute de données expérimentales, les caractéristiques manquantes, comme l'évolution des coefficients de dilatation thermique en fonction de la température, ont été prises dans la littérature en se rapprochant au mieux du béton de Holst (Diederichs et al., 1992 ; Bazant et al., 1996). Elles sont données dans les tableaux 2 et 3 , où $E$ est le module de Young, $v$ le coefficient de Poisson, $F_{t}$ la résistance à la traction, $F_{c}$ la résistance en compression, $G_{f}$ l'énergie de fissuration et $\alpha_{t h}$ le coefficient de dilatation thermique.

\begin{tabular}{|l|l|l|l|l|l|}
\hline & $\mathrm{E}(\mathrm{MPa})$ & $v$ & $\mathrm{~F}_{\mathrm{t}}(\mathrm{MPa})$ & $\mathrm{Fc}(\mathrm{MPa})$ & $\left.\mathrm{G}_{\mathrm{f}} \mathrm{N} / \mathrm{mm}\right)$ \\
\hline Pâte de ciment & 20000 & 0,20 & 4 & 70 & 0,05 \\
\hline Granulats & 80000 & 0,28 & 10 & 150 & 0,15 \\
\hline
\end{tabular}

Tableau 2. Caractéristiques mécaniques de la matrice cimentaire et des granulats

\begin{tabular}{|l|l|l|l|}
\hline \multicolumn{2}{|c|}{ Pâte de ciment } & \multicolumn{2}{c|}{ Granulats } \\
\hline $\mathrm{T}\left({ }^{\circ} \mathrm{C}\right)$ & $\alpha_{\text {th }}\left({ }^{\circ} \mathrm{C}^{-1}\right)$ & $\mathrm{T}\left({ }^{\circ} \mathrm{C}\right)$ & $\alpha_{\text {th }}\left({ }^{\circ} \mathrm{C}^{-1}\right)$ \\
\hline 20 & $10 \mathrm{e}-6$ & 20 & $3 \mathrm{e}-6$ \\
\hline 120 & $15 \mathrm{e}-6$ & 200 & $9 \mathrm{e}-6$ \\
\hline 400 & $-5 \mathrm{e}-6$ & 500 & $21 \mathrm{e}-6$ \\
\hline 1200 & $-25 \mathrm{e}-6$ & 800 & $57 \mathrm{e}-6$ \\
\hline
\end{tabular}

Tableau 3. Coefficient de dilatation thermique de la pâte et des granulats
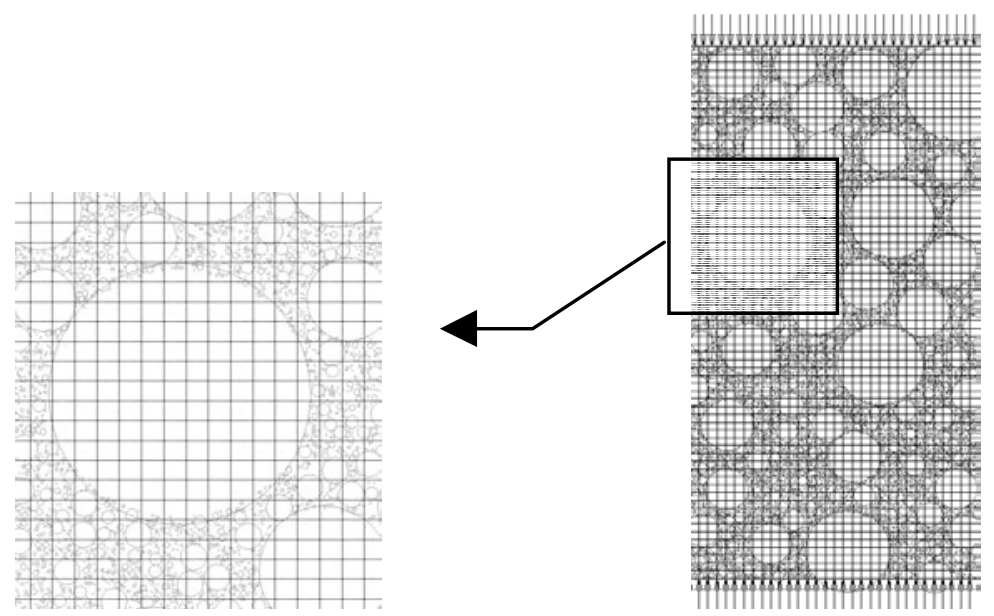

Figure 5. Maillage axisymétrique et conditions aux limites adoptés pour la simulation des essais de Holst : 2176 éléments QUAD4, charge répartie appliquée sur la partie supérieure égale à 0, 20\%, $40 \%$ ou $60 \%$ de la résistance en compression $F_{c}$, puis montée en température jusqu'à $500{ }^{\circ} \mathrm{C}$ avec une vitesse de $4,98^{\circ} \mathrm{C} / \mathrm{min}$ 


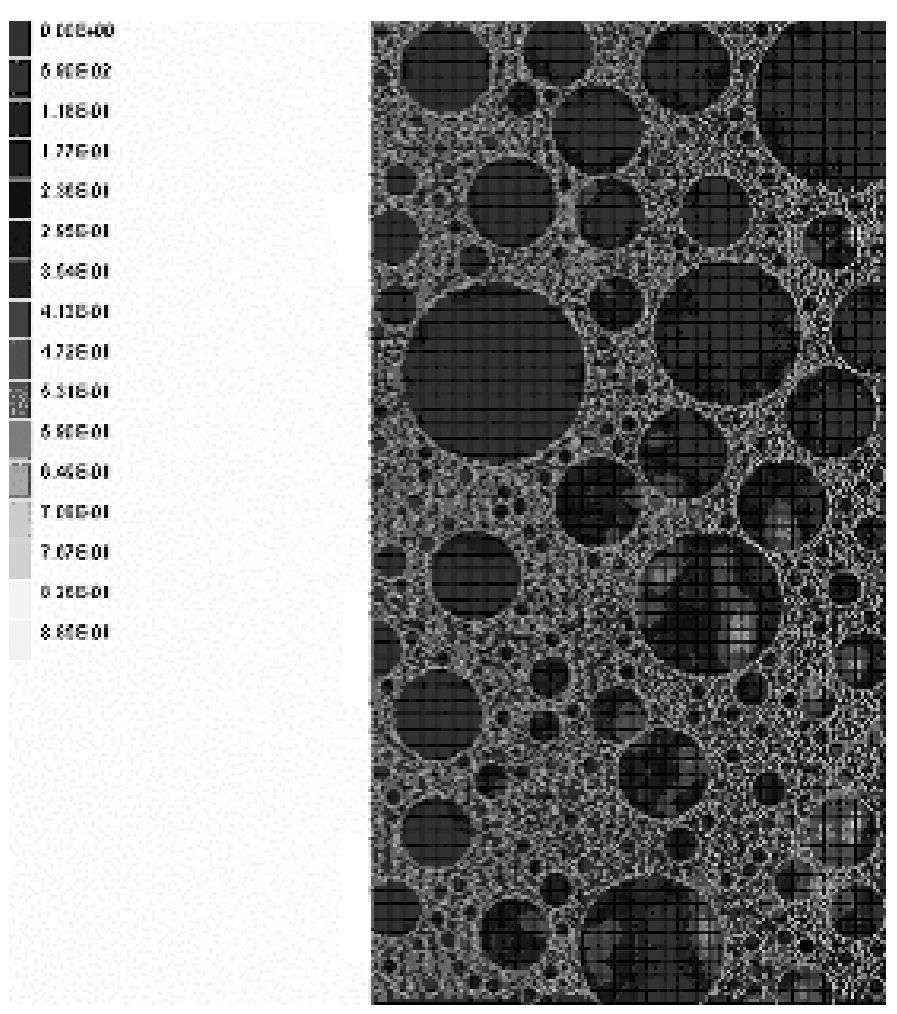

(a)

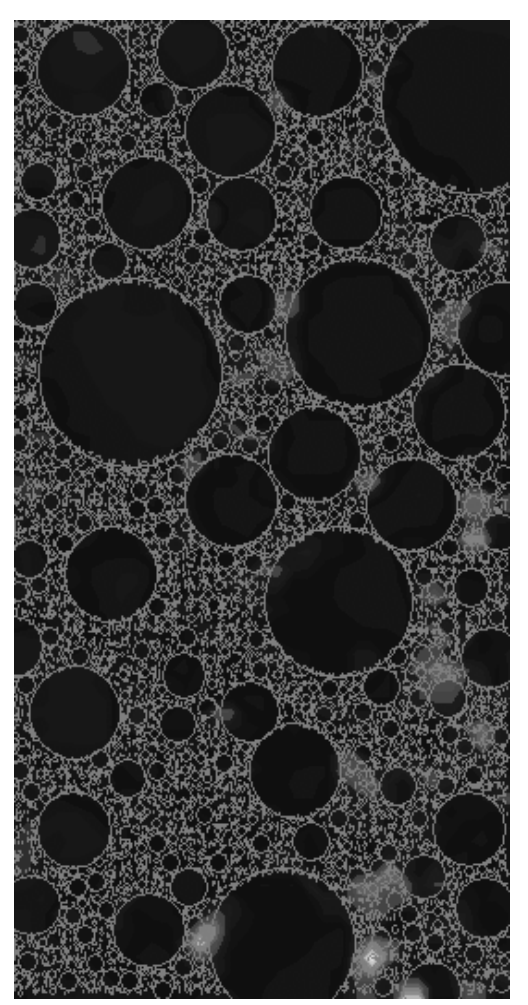

(b)

Figure 6. Endommagements déviatorique (a), et sphérique (b) à $120{ }^{\circ} \mathrm{C}$, localisés essentiellement dans la matrice cimentaire et aux interfaces matrice-granulat

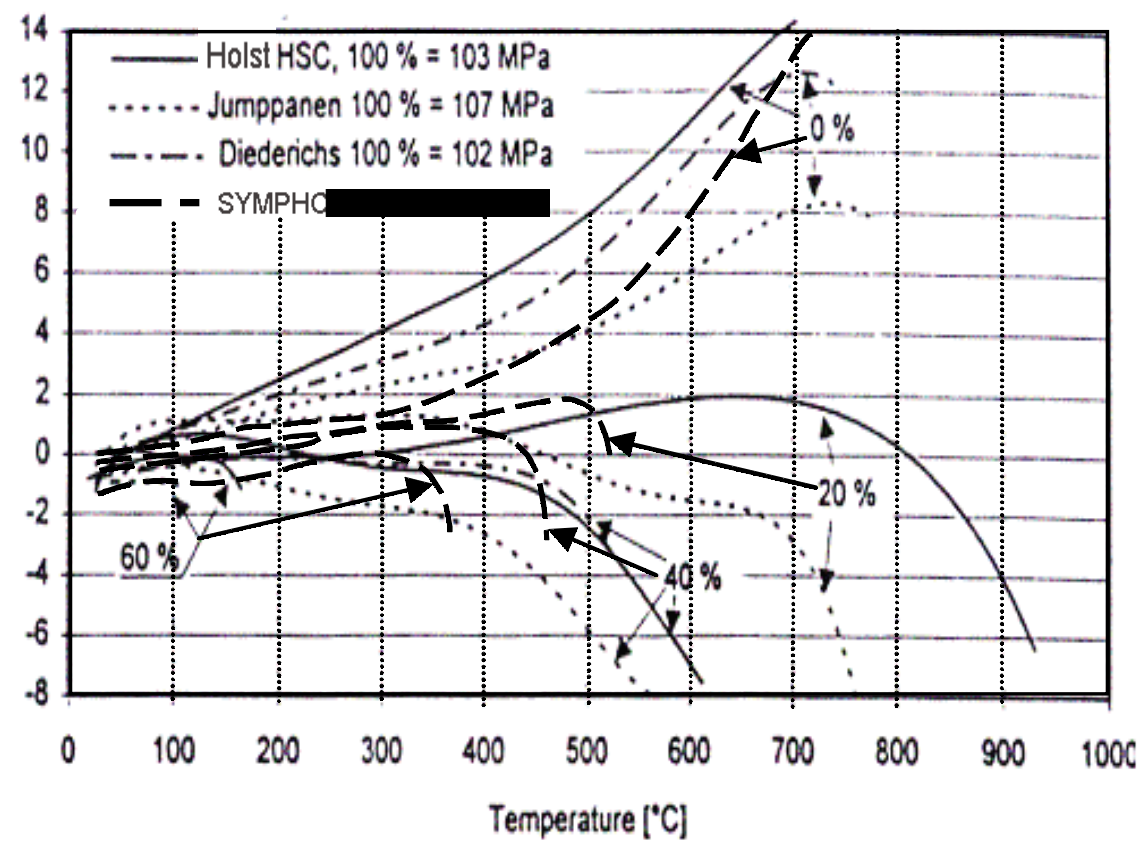

Figure 7. Comparaison essais calculs de l'évolution de la déformation thermique sous charge en fonction de la température pour différents niveaux de chargement $(0,20,40$ et $60 \%)$ 
Dans les modèles macroscopiques, l'endommagement obtenu pour ce type d'essais est souvent très faible, alors qu'à l'échelle des constituants, dans certaines phases du matériau, il peut être très important. L'approche micromécanique Béton Numérique permet de bien capter cet endommagement local (figure 6). Les résultats des simulations ont été comparés aux résultats d'essais de Holst (Holst, 1994) et à ceux de (Diederichs et al., 1992). Les simulations donnent une bonne tendance de l'évolution de la déformation thermique sous charge en fonction de la température et cela pour les différents niveaux de chargement, (figure 7). Notons que les résultats en termes d'endommagement thermique et de déformations sont très sensibles aux variations des coefficients de dilatation thermique de la matrice cimentaire et des granulats. Des données plus précises sur ces paramètres conduiraient probablement à de meilleurs résultats.

\section{Conclusion}

Une nouvelle approche pour la modélisation de l'endommagement des bétons à haute température a été présentée. Celui-ci, noté "endommagement thermique », a été décomposé en un endommagement d'origine mécanique dû aux déformations (macroscopiques et microscopiques) gênées et un endommagement physicochimique (non accompagné de déformations) dû aux transformations physicochimiques de la matière.

Pour montrer l'effet structure d'un échantillon en béton chauffé et simultanément sollicité en compression, des simulations ont été menées avec le modèle d'endommagement thermique à l'échelle des constituants du béton, générés avec le Béton Numérique. Ces simulations permettent de reproduire l'évolution de la déformation thermique sous charge en fonction de la température, constatée expérimentalement et montrent que celle-ci est due, en grande partie, à l'endommagement du matériau à haute température. Celui-ci est la combinaison d'une partie mécanique à l'échelle des constituants due à la différence de dilatation entre la pâte de ciment et les granulats lors de la montée en température et d'un endommagement dû aux transformations physicochimiques de la pâte de ciment. En d'autres termes, la montée en température endommage le matériau et augmente donc considérablement la déformation mécanique engendrée par la charge appliquée, même si celle-ci ne représente que 20 ou $40 \%$ de la résistance du matériau à froid.

\section{Bibliographie}

Bazant Z. P., Kaplan M., "Concrete at high temperature: material behaviour and mathematical modelling", London lonman concerte design and conconstruction series, 1996.

Diederichs U., Jumpanen U.-M., Penttala V., "Behaviour of high temperatures", Espoo 1989, Helsinki University of technology, Department of Structural Engineering, Report 92, 1992, p. 723. 
Grondin F., Dumontet H., Ben Hamida A., Mounajed G., Boussa H., "The numerical homogenization of the concrete behavior", XXI International Congress of Theoretical and Applied Mechanics, August 15-21, Warsaw, Poland, 2004.

Hansen T. C., Eriksson L., "Temperature change on behaviour of cement past, mortar and concrete under load", Journal of American Concrete Institute, 1966, vol. 63, n 4, 1966, p. 489-504.

Heinfling G., Reynouard J.-M., Merabet O., Duval C. A., "Thermo-elastic-plastic model for concrete at elevated temperatures including cracking and thermo-mechanical interaction strains", Owen D. R., Oñate E., Hinton E. (ed.), Computational plasticity: fundamentals and applications, vol. 2. Barcelona, Spain, CIMNE, 1997, p. 1493-8.

Hillerborg A., Modeer M., Petersson P. G., "Analysis of crack formation and crack growth in means by fracture mechanics and finite elements", Cement and concrete research, vol. 6, 1976, p. 773-786.

Holst F., "Mechanical properties of high strength concrete at high temperatures", High Performance Concrete, Interim Report, M7:2, Division of Building Materials, LTH, Lund, 1994.

Khoury G. A., Grainger B N., Sulivan G. P. E., "Transient thermal strain of concrete: litterature review, conditions with specimen and behaviour of individual constituents", Magazine of concrete research, $\mathrm{n}^{\circ} 132,1985$, p. 131-144.

Menou A., Etude du comportement thermomécanique des bétons à haute température. Approche multi-échelles de l'endommagement thermique, Thèse de doctorat de l'Université de Pau et des Pays de l'Adour, 2004.

Mounajed G., Code de calcul Général aux éléments Finis SYMPHONIE, http://mocad.cstb.fr, Paris, France, 1991.

Mounajed G., «Exploitation du nouveau modèle Béton Numérique dans Symphonie: concept, homogénéisation du comportement thermomécanique des BHP et simulation de l'endommagement thermique », Cahiers du CSTB, n 3421, 2002.

Mounajed G., Ung Quoc H., Boussa H. «Elaboration d'un nouveau modèle d'endommagement dans le code de calcul SYMPHONIE-CSTB», Revue française de génie civil, Hermès, vol. 7, n 5, 2003a, p. 679-689.

Mounajed G., Menou A., Boussa H., Pineaud A., Remond S., Cabrillac R. «Comportement au feu des bétons. Approche multi-échelles de l'endommagement thermique et identification expérimentale des paramètres ", Revue française de génie civil, Hermès, vol. $7, n^{\circ} 10,2003$ b, p. 1299-1323.

Mazars J., Application de la mécanique de l'endommagement au comportement non linéaire et à la rupture du béton de structure, Thèse de Doctorat de l’Université Paris 6, 1984.

Nechnech W., Meftah F., Reynouard J. M., "An elasto-plastic damage model for plain concrete subjected to high temperatures", Engineering Structures, 24, 2002, p. 597-611.

Schneider U., "Concrete at high temperatures; a general review", Fire Safety Journal, 13, p. $55,1988$. 
Ung Quoc H., Théories de dégradation du béton et développement d'un nouveau modèle d'endommagement en formulation incrémentale et tangente, Thèse de doctorat de l'ENPC, 2003. 